\title{
Nivel de conocimiento, actitud y experiencias de los profesionales de la salud respecto de la lactancia y la leche materna en una ciudad de Turquía: Estudio transversal
}

\author{
Knowledge level, attitude and own experience of health \\ professionals about breastfeeding and breast milk in a city of \\ Turkey: Cross-sectional study
}

\author{
Dra.Aylin Baydar Artantas ${ }^{a}$, Dra. Burcu Kayhan Tetis ${ }^{b}$, Enf. Melek Kıllc ${ }^{c}$, \\ Dra. Irep Karatas Eray ${ }^{a}$, Nutric. Nazan Cetin ${ }^{d}$, Partera Sinem Güneye, \\ Dr. Dogan Akdogan ${ }^{d}$,Dra. Umut Domac ${ }^{d}$, Prof. Yusuf Ustuf y Prof. Mehmet Ugurluf
}

a. Hospital de Formación e Investigación Atatürk de Ankara, Departamento de Medicina General.

b.İnönü Üniversitesi, Facultad de Medicina, Departamento de Medicina General.

c. Ministerio de Salud de Turquía.

d. Doctorado de Salud Pública de Ankara.

e. Hospital de Formación e Investigación Zekai Tahir Burak.

f. Yıldırım Beyazıt Üniversitesi, Facultad de Medicina, Departamento de Medicina General.

Correspondencia: Dra. Aylin Baydar Artantas: draylinbaydar@yahoo.com

Financiamiento:

Ninguno.

Conflicto de intereses: Ninguno que declarar.

Recibido: 30-3-2016 Aceptado: 19-8-2016 Publicado Primero en Internet: 14-10-2016

\section{RESUMEN}

Introducción. El consenso mundial acerca de la lactancia materna es que el bebé debe ser exclusivamente amamantado hasta los seis meses de vida. A pesar de ello, la tasa de lactancia materna exclusiva es baja durante los primeros seis meses de vida en muchos países, incluida Turquía. El objetivo de este estudio fue evaluar el conocimiento, las actitudes y las experiencias de los profesionales sanitarios en relación con la lactancia y la leche materna en una ciudad metropolitana de Turquía.

Materiales y métodos. Esteestudio sellevó a cabo entrejulio de 2012y diciembre de2013. Los sujetos del estudio fueron profesionales de la salud que trabajaban enhospitales universitarios, hospitales de formación e investigación, hospitales públicos y privados, centros de servicios para niños, adolescentes, mujeres y de servicios de salud reproductiva, centros de salud general y centros de salud comunitarios ubicados en Ankara. Los datos se recogieron por medio de un cuestionario. El programa SPSS 11.5 se utilizó para el análisis estadístico.

Resultados. En total, 715 personas participaron en este estudio. Entre los que tenían hijos propios, el $3,4 \%(n=16)$ no había amamantado a sus bebés y el $7,6 \%(\mathrm{n}=36)$ había amamantado a sus bebés hasta el segundo año de vida. Se observó que el nivel de conocimiento sobre la lactancia y la lecha materna era deficiente en el $28,4 \%$ de los participantes, promedio en el $43,1 \%$ y adecuado en el $28,5 \%$. Conclusión. Este estudio refleja la falta de conocimiento acerca de la lactancia y la leche materna entre los profesionales de la salud de Turquía. Si bien la gran mayoría de los participantes están de acuerdo con la recomendación de la OMS acerca de la duración de la lactancia materna, el porcentaje de profesionales de la salud que amamantaron durante el período recomendado fue muy bajo. Palabras clave: Leche materna, lactancia materna, profesionales de la salud, Turquía.

http:/ /dx.doi.org/10.5546/aap.2016.514 Texto completo en inglés: http://dx.doi.org/10.5546/aap.2016.eng.514

\section{INTRODUCCIÓN}

La Organización Mundial de la Salud (OMS) y el Fondo para la Infancia de las Naciones Unidas (UNICEF) recomiendan la lactancia exclusiva hasta los seis meses de vida. ${ }^{1}$

En Turquía, también se aceptan y respaldan estas recomendaciones.

En los estudios realizados en países en vías de desarrollo se demostró que el riesgo de mortalidad entre los lactantes que no reciben leche materna es entre seis y diez veces mayor que entre los que sí consumen leche materna. ${ }^{2,3}$ La tasa de neumonía y diarrea, dos de las causas más frecuentes de mortalidad entre los niños menores de cinco años, son mayores y tienen un peor pronóstico entre los lactantes que no reciben leche materna. ${ }^{4,5}$ Los efectos a largo plazo de la lactancia materna incluyen un riesgo menor de obesidad y puntajes más elevados en la función cognitiva entre quienes recibieron leche materna. ${ }^{6}$

La OMS y UNICEF recomiendan que la lactancia materna comience en la primera hora posparto. ${ }^{7} \mathrm{El}$ calostro protege a los lactantes contra las infecciones y también regula las funciones del sistema digestivo del bebé. ${ }^{6}$ Asimismo, permite que la madre entre en contacto con su niño lo más pronto posible, lo que ayuda a fortalecer el vínculo entre ambos.

A pesar de los beneficios de la lactancia materna, la tasa de lactancia exclusiva entre los lactantes hasta los 
seis meses es de aproximadamente el $36 \% .^{7}$ En 2012, la Asamblea Mundial de la Salud declaró, entre los seis objetivos mundiales, el aumento de las tasas de lactancia materna exclusiva en los primeros seis meses de vida al menos hasta el $50 \%$ hacia 2025. En realidad, la tasa de lactancia materna exclusiva aumentó de aproximadamente el $14 \%$ al $38 \%$ entre 1985 y 1995, pero luego disminuyó en muchas regiones del mundo. ${ }^{8,9} \mathrm{La}$ lactancia materna es frecuente en Turquía, pero la tasa de lactancia materna exclusiva disminuye del $68,9 \%$ al 23,6\% entre los cuatro y los seis meses de vida. Asimismo, solamente el 39\% de los lactantes son amamantados en la primera hora después del parto y el $27 \%$ de los bebés no son amamantados en las primeras 24 horas. ${ }^{10}$ Las creencias y los conocimientos incorrectos sobre la lactancia materna, el acceso inadecuado a los servicios sanitarios y a los profesionales de la salud y el poco respaldo brindado por los profesionales de la salud podrían ser las causas de dichos resultados desfavorables.

Este estudio se llevó a cabo para evaluar el nivel de conocimiento, las actitudes y las experiencias de los profesionales de la salud acerca de la lactancia y la leche materna en una ciudad metropolitana de Turquía.

\section{MATERIALES Y MÉTODOS}

Este estudio transversal se llevó a cabo entre el 1 de julio de 2012 y el 31 de diciembre de 2013 en los hospitales y centros de atención primaria de Ankara, la capital de Turquía.

Se planificó la inclusión de los profesionales de la salud que las mujeres consultan con mayor frecuencia antes y después del parto. Se incluyó a especialistas y residentes de ginecología y obstetricia, pediatría y medicina general, y a médicos de cabecera, enfermeras y parteras. Se seleccionaron 29 hospitales entre los 90 que existen en Ankara. Se tomó en cuenta la cantidad de partos en cada hospital, y se estableció el criterio de más de 500 partos mensuales en promedio como criterio de selección. Se obtuvo la cantidad de profesionales de la salud (ginecobstetras, pediatras, médicos generales, médicos de cabecera, enfermeras y parteras) que trabajan en estos 29 hospitales y en los centros de atención primaria de Ankara de la Unidad de Personal del Ministerio de Salud. Según los datos del Ministerio de Salud de Turquía, la escala del estudio se estableció en 4985 personas. El objetivo era alcanzar al 10\% de estas personas $\mathrm{y}$, considerando los datos ausentes, se seleccionó a unas 750 para participar en el estudio. Los siguientes fueron los criterios de inclusión: ser un profesional de la salud, estar trabajando activamente en estas instituciones sanitarias y completar el cuestionario.

El cuestionario (Anexo electrónico) se utilizó para la recolección de datos e incluía 29 preguntas: ocho sobre características sociodemográficas, 16 sobre el nivel de conocimiento acerca de la lactancia y la leche materna, una sobre la información brindada durante la práctica diaria y cuatro sobre la capacitación recibida sobre lactancia materna. Se categorizó a los participantes según su nivel de conocimiento y se los dividió en tres grupos de conocimiento: deficiente, promedio y adecuado. La categorización de los grupos dependía de la cantidad de respuestas correctas. Se realizó un estudio previo con 92 personas para revisar y finalizar el cuestionario. Los participantes completaron todos los cuestionarios bajo observación directa en su lugar de trabajo.

Se obtuvo la aprobación del Comité de Etica de Ensayos Clínicos No Farmacológicos del Hospital de Formación e Investigación Atatürk.

\section{Análisis estadístico}

Las variables numéricas descriptivas separadas se expresaron como media \pm desviación estándar o mediana (mínimo-máximo). Las variables categóricas se expresaron como cantidad de participantes y porcentajes, y se evaluaron con la prueba de $\chi^{2}$ de Pearson y la prueba exacta de Fisher. Los resultados con un valor de $p<0,05$ se consideraron estadísticamente significativos. Los datos se analizaron con el paquete de software SPSS 11.5 para M.S. Windows.

\section{RESULTADOS}

En total, 715 profesionales de la salud participaron en este estudio. La edad de los participantes varió entre los 17 y los 62 años, con una media de 35,6 6 7,8 años. En la Tabla 1, se presentan otras características de los participantes.

El 70,1\% (n= 501) tenían al menos un hijo. La mediana de la duración de la lactancia fue un año. Además, el 3,2\% $(n=16)$ de los participantes que tenían hijos nunca habían amamantado a su bebé.

Una clara mayoría de los participantes $(94,9 \%)$ recomendaban la lactancia materna exclusiva durante los primeros seis meses de vida. Para el período posterior al inicio de la alimentación complementaria, la mayoría de los participantes $(84,8 \%)$ recomendaban continuar con la lactancia materna hasta los dos años de vida, mientras 
TABLA 1. Características de los profesionales de la salud que participaron en el estudio $(n=715)$

\begin{tabular}{lcc}
\hline & $\mathbf{n}$ & $\%$ \\
\hline Sexo & & \\
Femenino & 587 & 82,1 \\
Masculino & 128 & 17,9 \\
Profesión & & \\
Especialista en ginecología y obstetricia & 7 & 1,0 \\
Residente de ginecología y obstetricia & 18 & 2,5 \\
Especialista en pediatría & 2 & 0,3 \\
Residente de pediatría & 9 & 1,3 \\
Especialista en medicina general & 11 & 1,5 \\
Residente de medicina general & 53 & 7,4 \\
Médico de cabecera & 74 & 10,3 \\
Partera/enfermera & 447 & 62,5 \\
Otra & 94 & 13,2 \\
Institución & & \\
Hospital universitario & 12 & 1,7 \\
Hospital de formación e investigación & 204 & 28,5 \\
Hospital público & 149 & 20,8 \\
Centro de salud general & 179 & 25,0 \\
Centro de salud comunitario & 121 & 16,9 \\
Centros de servicios para niños, adolescentes, & \\
mujeres y de servicios de salud reproductiva & 16 & 2,3 \\
Hospital militar & 34 & 4,8 \\
Experiencia profesional & & \\
De 1 a 5 años & 173 & 24,2 \\
De 6 a 10 años & 111 & 15,5 \\
De 11 a 15 años & 109 & 15,2 \\
De 16 a 20 años & 164 & 22,9 \\
De 21 a 25 años & 95 & 13,3 \\
>25 años & 55 & 7,7 \\
\hline & & \\
& & \\
& & \\
& &
\end{tabular}

que el 10,1\% recomendaban la lactancia materna hasta el año de vida. Casi todos los participantes $(97,5 \%)$ fomentaban la lactancia del bebé en los primeros 30 minutos después del parto.

E1 3,6\% de los participantes tenían conocimientos precisos sobre las enfermedades cuyo riesgo se reduce con la lactancia materna durante el período neonatal. Las alergias y la obesidad fueron las dos afecciones sobre las cuales los participantes tenían un conocimiento preciso (79\% y 75\%, respectivamente). El 96,6\% de los participantes declararon que la lactancia materna tenía un efecto protector contra el cáncer de mama.

Más de la mitad de los participantes creían, erróneamente, que algunas afecciones en los pezones y la mama eran un obstáculo para la lactancia materna. Casi la mitad de los participantes $(59,9 \%)$ indicaron que la leche materna no sería suficiente en el caso de mellizos y que se debía usar leche maternizada.

En general, el nivel de conocimiento acerca de la lactancia y la leche materna era "deficiente" en el $28,4 \%$ de los participantes, "promedio" en el 43,1\% y "adecuado" en el 28,5\% (Tabla 2).

Entre los profesionales incluidos en el estudio, el 60,7\% declararon que brindaban activamente asesoramiento a las madres en relación con la lactancia y la leche materna en la práctica diaria.

TABLA 2. Nivel de conocimiento de los profesionales de la salud acerca de la lactancia y la leche materna

\begin{tabular}{lccc}
\hline Profesión & Deficiente & Promedio & \multicolumn{2}{c}{$\begin{array}{c}\text { Adecuado } \\
\mathbf{n}(\%)\end{array}$} \\
\hline Médico de cabecera $(\mathrm{n}=74)$ & $\mathbf{n}(\%)$ & $24(32,5)$ & $36(48,6)$ \\
Ginecobstetra $(\mathrm{n}=25)$ & $14(18,9)$ & $11(44,0)$ & $6(24,0)$ \\
Pediatra $(\mathrm{n}=11)$ & $8(32,0)$ & $6(54,5)$ & $2(18,2)$ \\
Médico general $(\mathrm{n}=64)$ & $3(27,3)$ & $29(45,3)$ & $16(25,0)$ \\
Partera/enfermera $(\mathrm{n}=447)$ & $19(29,7)$ & $197(44,1)$ & $133(29,7)$ \\
\hline
\end{tabular}

TABLA 3. Capacitación de los participantes sobre la lactancia y la leche materna

\begin{tabular}{lcc}
\hline & $\mathbf{n}$ & $\%$ \\
\hline ¿Alguna vez asistió a un programa de capacitación sobre lactancia y leche materna? & & 702 \\
$\quad$ Sí & 213 & 70,2 \\
No & & 29,8 \\
¿Cuándo y dónde asistió a dicho programa de capacitación? & 161 & 32,1 \\
$\quad$ Durante mi carrera y especialización & 348 & 69,3 \\
En el lugar de trabajo & 38 & 7,6 \\
En el hospital donde nació mi bebé & 26 & 5,2 \\
Durante los controles del embarazo & 39 & 7,8 \\
Otro & & \\
¿Requiere un programa de capacitación sobre lactancia materna? & 293 & 41,0 \\
Sí & 404 & 56,5 \\
No & & \\
\hline
\end{tabular}


Se observó que el 76,3\% de las enfermeras y parteras, el $73 \%$ de los médicos de cabecera, el $72 \%$ de los ginecobstetras, el $63,6 \%$ de los pediatras y el $51,6 \%$ de los médicos generales habían recibido capacitación sobre lactancia materna en algún momento del ejercicio de su carrera. En la Tabla 3, se describe la experiencia en capacitación de los participantes.

\section{DISCUSIÓN}

La leche materna es la principal fuente de alimento de los lactantes, ya que contiene nutrientes importantes. ${ }^{11}$ Desafortunadamente, en la mayoría de los países, no se amamanta adecuadamente a muchos lactantes. A pesar de las recomendaciones sobre la lactancia materna, excepto en Alemania, Noruega y Escandinavia, la tasa de lactancia materna a nivel mundial es baja. ${ }^{12-14}$ La decisión de la madre no es el único factor que influye en la finalización anticipada de la lactancia materna; otros factores incluyen dolor en los pezones, congestión mamaria, agitación del lactante, dudas acerca de tener suficiente leche materna y las creencias acerca de la finalización anticipada de la lactancia a nivel social. Otra de las razones principales es la falta de conocimiento de los profesionales de la salud a la hora de abordar los problemas relacionados con la lactancia materna. ${ }^{15}$ Este estudio se diseñó para establecer el nivel de conocimiento de los profesionales de la salud acerca de la lactancia y la leche materna y es el primero de este tipo en Turquía.

Los investigadores observaron que el problema más frecuente con la lactancia materna exclusiva es el trabajo de las madres. Otros factores incluyen afecciones médicas maternoinfantiles, creencias diversas acerca de la nutrición infantil y factores socioculturales. ${ }^{16}$ En un estudio realizado en Uganda, se observó que los bebés no estaban adecuadamente alimentados, ni con leche materna ni con alimentos complementarios. La falta de conocimiento se mencionó como el principal factor del problema. Las condiciones de trabajo estresantes, ya sea dentro o fuera del hogar, son el principal problema relacionado con la lactancia materna exclusiva durante los primeros seis meses. También se citó que los profesionales de la salud pasaron por alto muchas oportunidades para brindar la información correcta relacionada con la nutrición infantil a las madres. ${ }^{17}$

En estudios realizados en diferentes países acerca de los profesionales de la salud, se observó que las recomendaciones de la OMS sobre la duración de la lactancia materna no se brindan ni fomentan adecuadamente. El 21\% de los participantes en un estudio llevado a cabo con enfermeras y parteras respaldaron la lactancia materna durante los primeros seis meses del lactante y el 33\% la fomentaron hasta los 24 meses de vida. ${ }^{18}$ En diferentes estudios, el 35\% de los participantes respaldaron la lactancia materna exclusiva durante los primeros seis meses de vida, y el $27,5 \%$ y el $36 \%$ fomentaban continuar con la lactancia hasta los dos años. ${ }^{19,20}$ Ramakrishnan y cols., observaron que el 32,7\% de las madres atendidas por obstetras y el $44,8 \%$ de las madres atendidas por pediatras tenían la percepción de que el profesional de la salud les había recomendado la lactancia materna exclusiva durante el primer mes de vida solamente. ${ }^{21}$ En nuestro estudio, se observó que la mayoría de los participantes $(94,9 \%)$ respaldaban la lactancia materna exclusiva durante los primeros seis meses y que el $84,8 \%$ fomentaban continuar con la lactancia materna hasta los dos años. Si bien la mayoría de los participantes parecían respaldar las políticas de la OMS respecto de la lactancia materna, se halló que solamente el 7,6\% habían continuado con el amamantamiento de sus hijos hasta los dos años de vida. En otro estudio, se observó que si bien los médicos respaldan la lactancia materna, no podían recordar el tiempo durante el cual habían amamantado a sus propios hijos. Nuevamente, declararon que el principal factor que interrumpía la lactancia materna era el trabajo. ${ }^{22}$

Existen muchos estudios llevados a cabo con médicos para evaluar su nivel de conocimiento acerca de la lactancia y la leche materna. En uno de los estudios, la tasa de respuestas correctas a las preguntas sobre este tema fue del $67,8 \%$ entre los pediatras, del $64,3 \%$ entre los médicos generales, del $72,7 \%$ entre los residentes de pediatría y del $66,8 \%$ entre los residentes de medicina general. ${ }^{23}$ En nuestro estudio, se observó que los médicos de cabecera eran quienes obtenían más respuestas correctas a las preguntas sobre su conocimiento acerca de la lactancia y la leche materna, y quienes brindaban más asesoramiento en su práctica diaria. Desafortunadamente, no es posible comentar acerca del nivel de conocimiento de los otros profesionales, especialmente los pediatras y los ginecobstetras, debido a su participación limitada. En diferentes estudios, la tasa de asesoramiento sobre lactancia materna entre los médicos fue del $20 \%$ y el $39 \% .{ }^{24,25}$ El resultado de un estudio realizado en Australia demostró que, durante la atención prenatal, el 32,6\% de las pacientes 
recibieron asesoramiento de su ginecobstetra y el 18,4\% habían recibido asesoramiento de su médico general acerca de la lactancia materna. ${ }^{26}$ Un estudio realizado en Turquía indicó que, entre las mujeres con hijos, el 62,2\% habían recibido información de un profesional de la salud. ${ }^{27} \mathrm{El} 63,3 \%$ de los participantes de nuestro estudio declararon que brindaban asesoramiento activamente en su práctica diaria. En otro estudio llevado a cabo en Turquía, la tasa fue similar. ${ }^{28}$ Si bien la tasa de asesoramiento parece alta en Turquía, se analizará la eficacia de los servicios de asesoramiento. Especialmente, el inicio del asesoramiento durante los controles prenatales y su continuación después del parto podrían ser eficaces sobre el inicio y la continuación de la lactancia materna. En una revisión Cochrane, se estableció que el asesoramiento profesional prolonga la duración general de la lactancia materna y la duración de la lactancia materna exclusiva. Debería brindarse apoyo social como parte de la rutina de los centros de atención de la salud según las necesidades de las personas que viven en esa área. También se ha establecido que el contacto personal es mucho más eficaz que el contacto telefónico a la hora de brindar asesoramiento. ${ }^{15}$

En Turquía, el Ministerio de Salud organiza programas de capacitación sobre lactancia y leche materna destinados a los profesionales de la salud. Debido a la cantidad limitada de recursos humanos de los programas, no se alcanza a una cantidad suficiente de profesionales de la salud. En otro estudio llevado a cabo en Turquía, se observó que el $44 \%$ de los profesionales de la salud habían participado en dicha capacitación. ${ }^{29}$ En nuestro estudio, la tasa fue más alta. Una de las causas de esto podría ser el lugar donde se llevó a cabo el estudio. Ankara es la capital del país, por lo que es más fácil acceder a los programas de capacitación. De este modo, el lugar donde se hizo el estudio podría considerarse como una limitación.

Para fomentar una lactancia materna correcta y adecuada, es necesario brindar conocimiento actualizado y adecuado a la comunidad en primer lugar. Luego, podría implementarse dicho conocimiento en la práctica profesional. Esta cascada de transmisión del conocimiento e implementación práctica requiere de adecuados servicios de asesoramiento y capacitación comunitaria. Por lo tanto, es sumamente importante que los profesionales de la salud que tienen mayor contacto con las mujeres, antes y después del parto, reciban capacitación adecuada sobre este tema. En un estudio realizado en Irlanda, se observó que la cantidad de profesionales de la salud que recibieron capacitación sobre lactancia y leche materna es insuficiente y que los profesionales de la salud no tienen la capacidad para brindar asesoramiento y respaldo debido a la falta de tiempo. Por lo tanto, deben propagarse métodos alternativos entre las mujeres, como el "apoyo entre pares". ${ }^{30}$ Los médicos deben apuntar a aprovechar cada oportunidad para brindar asesoramiento y asistencia a las mujeres y la comunidad. En este sentido, los centros de atención primaria son lugares excelentes donde los profesionales de la salud tienen la posibilidad de brindar estos servicios. Los centros de atención primaria son diferentes de las instituciones de mayor complejidad dado que allí asisten no solamente pacientes sino también personas sanas. En este estudio, se observó que los médicos de cabecera, que trabajan en los centros de atención primaria, asumen una mayor responsabilidad sobre este tema en comparación con el resto de los médicos. Sin embargo, en otros estudios, se observaron resultados diferentes. En Canadá, la mitad de los médicos consideraban que la evaluación de la lactancia materna no es responsabilidad de los médicos que trabajan en los centros de atención primaria. ${ }^{23}$

La mayoría de los estudios sobre este tema tenían por objetivo evaluar la actitud y el enfoque de ciertos grupos de profesionales, como los ginecobstetras, los pediatras, los médicos generales, las enfermeras y las parteras. Esto podría ser producto de la aceptación mundial de que los profesionales de este grupo son quienes tienen mayor contacto con las mujeres, antes y después del parto.

El inicio temprano de la lactancia materna, la lactancia materna exclusiva durante los primeros seis meses y la continuación de la lactancia materna junto con la alimentación complementaria hasta los dos años son las metas relacionadas con lactancia en la mayoría de los países, incluida Turquía. La capacitación a nivel comunitario y el incremento de los servicios de asesoramiento brindados por los profesionales de la salud son obviamente las principales herramientas para alcanzar dichas metas.

Además deberían investigarse todos los aspectos de los obstáculos de la lactancia materna. En Turquía, la licencia por maternidad cubre solamente 16 semanas (ocho antes del parto y ocho después). Existe la opción de trasladar cinco 
semanas al período posparto. ${ }^{31}$ Esto significa que una madre puede extender la licencia por maternidad solamente hasta los tres meses, por lo que es muy difícil que pueda amamantar exclusivamente a su bebé durante los primeros seis meses.

Los derechos de los trabajadores de un país, incluidas las licencias de quienes trabajan en instituciones públicas o privadas, están regulados en conjunto y por muchas instituciones, tales como ministerios, institutos de seguridad social o asistencia y sindicatos. De esta manera, estos derechos están relacionados directamente con la situación económica y el nivel de empleo de dicho país. Sin embargo, deberá considerarse esta cuestión desde la lógica de que la leche materna es la principal fuente de nutrientes de los recién nacidos y que solamente la madre puede brindársela al bebé.

Si bien este estudio tiene muchas limitaciones, tales como el autoinforme sobre las actitudes respecto del asesoramiento sobre la lactancia materna y la participación limitada de pediatras y ginecobstetras, este estudio brinda una perspectiva sobre el estado de los profesionales de la salud en relación con la lactancia materna. A fin de contribuir con la reformulación de las políticas y los planes relativos a este tema, se requieren estudios más integrales y deben determinarse explícitamente las barreras y los catalizadores de la lactancia materna y el asesoramiento al respecto.

\section{CONCLUSIÓN}

Este estudio refleja la falta de conocimiento acerca de la lactancia y la leche materna entre los profesionales de la salud en una ciudad de Turquía. El porcentaje de lactancia materna exclusiva entre los profesionales de la salud durante el período recomendado fue muy bajo. $\mathrm{n}$

\section{REFERENCIAS}

1. World Health Organization. Infant and young child feeding. Model Chapter for textbooks for medical students and allied health professionals. France; WHO,2009. [Acceso: 23 de agosto de 2016] Disponible en: http://apps.who. int/iris/bitstream/10665/44117/1/9789241597494_eng. pdf?ua $=1$

2. Effect of breastfeeding on infant and childhood mortality due to infectious diseases in less developed countries: a pooled analysis. WHO Collaborative Study Team on the Role of Breastfeeding on the Prevention of Infant Mortality. Lancet 2000;355(9202):451-5.

3. Bahl R, Frost C, Kirkwood BR, Edmond K. et al. Infant feding patterns and risks of death and hospitalization in the firsthalf of infancy: multicentre cohortstudy. Bull World Health Organ 2005;83(6):418-26.
4. De Zoysa I, Rea M, Martines J. Why promote breastfeeding in diarrhoeal disease control programmes? Health Policy Plan 1991;6(4):371-9.

5. Bachrach VR, Schwarz E, Bachrach LR. Breastfeeding and the risk of hospitalization for respiratory diseases in infancy: a meta-analysis. Arch Pediatr Adolesc Med 2003;157(3): 237-43.

6. World Health Organization. Media Centre. Infant and young childfeeding. [Acceso: 23 de agosto 2016] Disponible en: http://www.who.int/mediacentre/factsheets/fs342/en/

7. Samur G. Anne sütü. Ankara: Klasmat Press; 2008.P.9.

8. World Health Organization. Nutrition. WHA65/6. Comprehensive implementation plan on maternal, infant and young child nutrition. [Acceso: 28 de junio de 2014] Disponible en: http://www.who.int/nutrition/topics / wha_65_6/en/

9. UNICEF. Progress for Children. A World Fit for Children Statistical Review. New York, USA:UNICEF, 2007. [Acceso: 23 deagosto 2016] Disponible en: http:/ / www.unicef.org/ publications/files/Progress_for_Children_No_6_revised. pdf

10. Children's and women's nutritional status. En: Turkey Demographic and Health Survey 2008. Ankara, Turkey: Hacettepe University Institute of Population Studies, 2009:177-86. [Acceso: 23 de agosto 2016] Disponible en: http://www.hips.hacettepe.edu.tr/eng/tdhs08/TDHS2008_Main_Report.pdf

11. Cope MB, Allison DB. Critical review of the World Health Organization's (WHO) 2007 report on 'evidence of the long term effects of breastfeeding: systematic reviews and metaanalysis' with respect to obesity. Obes Rev 2008;9(6):594-605.

12. Cattaneo A, Yngve A, Koletzko G, Guzman LR. Protection, promotion and support of breastfeeding in Europe: current situation. Public Health Nutr 2005;8(1):31-46.

13. Cattaneo A. Protection, promotion and support of breastfeeding in Europe: ablueprint for action. Luxembourg: European Commission, Directorate Public Health and Risk Assesstment, 2004. [Acceso: 23 de agosto 2016] Disponible en: http:/ / ec.europa.eu/health/ph_projects / 2002/ promotion/fp_promotion_2002_frep_18_en.pdf

14. Nicoll A, Thayaparan B, Newell ML, Rundall P. Breast feeding policy, promotion and practice in Europe. Resultsof a survey with non-governmental organizations. J Nutr Environ Med 2002;12(3):255-64.

15. Renfrew MJ, McCormick FM, Wade A, Quinn B. et al. Support for healthy breastfeeding mothers with healthy term babies. Cochrane Database Syst Rev 2012;(5):CD001141.

16. Balogun OO, Dagvadorj A, Anigo KM, Ota E, et al. Factors influencing breastfeeding exclusivity during the first 6 months of life in developing countries: a quantitative and qualitative systematic review. Matern Child Nutr 2015;11(4):433-51.

17. Nankumbi J,MuliiraJK. Barriers to Infant and Child-feeding Practices: A Qualitative Study of Primary Care givers in Rural Uganda. J Health Popul Nutr 2015;33(1):106-16.

18. Dachew BA, BifftuBB. Breastfeeding practice and associated factors among female nurses and midwives at North Gondar Zone, Northwest Ethiopia: a crosssectional institution based study. Int Breastfeed J 2014;9:11.

19. Leviniene G, Petrauskiene A, Tamilevicience E, Kudzyte J, etal. The evaluation of knowledge and activities of primary health care professionals on promoting breastfeeding. Medicina (Kaunas) 2009;45(3):238-47.

20. OkoloSN, Ogbonna C. Knowledge, attitude and practice of health workers in keffilocal governmenthospitals regarding Baby-friendly hospital initiative(BFHI) practices. Eur JClin Nutr 2002;56(2):438-41. 
21. Ramakrishnan R, Oberg CN,Kirby RS. The Association between Maternal Perception of Obstetric and Pediatric Care Providers' Attitudes and Exclusive Breastfeeding Outcomes. J Hum Lact 2014;30(1):80-7.

22. Anchondo I, Berkeley L, Mulla ZD, Byrd T, et al. Pediatricians', Obstetricians', Gynecologists', and Family Medicine Physicians' Experiences with and Attitudes about Breast-Feeding. South Med J 2012;105(5):243-8.

23. Pound CM, Williams K, Grenon R, Aglipay M, et al. Breastfeeding Knowledge, Confidence, Beliefs, and Attitudes of Canadian Physicians. J Hum Lact 2014;11;30(3):298-309.

24. Nakar S, Peretz O, Hoffman R, Grossman Z, et al. Attitudes and knowledge on breastfeeding among pediatricians, family physicians, and gynecologists in Israel. Acta Pædiatr 2007;96(6):848-51.

25. DiGirolamo AM, Grummer-Strawn LM, Fein SB. Do Perceived Attitudes of Physicians and Hospital Staff Affect Breastfeeding Decisions? Birth 2003;30(2):94-100.
26. Walsh AD, Pincombe J, Henderson A. An Examination of Maternity Staff Attitudes Towards. Implementing Baby Friendly Health Initiative (BFHI) Accreditation in Australia. Matern Child Health J 2011;15(5):597-609.

27. Ünsal H, Atlıhan F, Özkan H, Targan , et al. Toplumda anne sütü verme e ilimi ve buna etki eden faktörler. Çocuk Sa lı 1 ve Hastalikları Dergisi 2005;48:226-33.

28. Kaya D, ve Pirinçci E. 0-24 Aylık Çocu u Olan Annelerin Anne Sütü ve Emzirme le lgili Bilgi ve Uygulamaları. TAF Prev Med Bull 2009;8(6):479-84.

29. Gonener HD, Balat A, Aydın N. Cocuk Sa lı1 le lgili Alanlarda Calı mayan Hekim ve Hem irelerin AnneSutune Yonelik Bilgi Durumları. ÜFN Hem Derg 2010;18 (3):128-35.

30. Whelan B, Kearney JM. Breast-feeding support in Ireland: a qualitative study of health-careprofessionals' and women's views. Public Health Nutr 2015;18(12):2274-82.

31. Labour act of Turkey. Law 4857, article 74 (june 10, 2003). [Acceso: 23 de agosto 2016] Disponible en: https: / www. ilo.org/dyn/natlex/docs/ELECTRONIC/64083/77276/ F75317864/TUR64083\%20English.pdf

\section{Juan B. Ursomarso}

Falleció el 6-9-2016

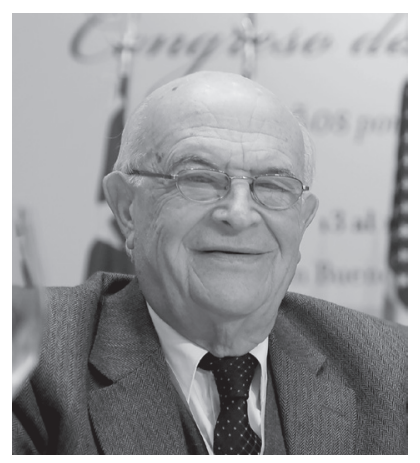

Con la ida de nuestro muy querido Chacho se fue un gran amigo y una excepcional persona. Hace ya muchos años nos conocimos en actividades relacionadas con la Sociedad Argentina de Pediatría, donde participaba con gran ahínco, entusiasmo y un alto compromiso. Siempre bregó en forma incansable para lograr mejoras en la atención de los niños y sus familias en su muy querida Mendoza, y progresivamente se convirtió en un gran hacedor de múltiples emprendimientos, medidas sanitarias, programas de educación médica y otros.

Entre esas actividades se destacan su participación decisiva en la construcción y puesta en marcha del Hospital Notti, su extraordinaria dedicación a la SAP que ubicó a la Filial Mendoza entre las más destacadas del país y la presidencia del Congreso Nacional de Pediatría en 1997, entre varios más. Asimismo, fue miembro de la Comisión Directiva en la Entidad Matriz y formó parte del grupo que elaboró los últimos cambios en los estatutos de nuestra sociedad.

Todos estos aspectos y muchos otros, lo situaron entre uno de los más destacados pediatras argentinos de los últimos años.

Finalmente, deseo enfatizar sus enormes atributos como persona, su conducta intachable, su humildad, su gran bonhomía, su notable sentido del humor, y podríamos continuar con una larga lista. La inmensa pena que me ocasiona su partida es mitigada por los recuerdos imborrables que persisten nítidamente en mi memoria y por la enorme fortuna de haber disfrutado de su muy cálida amistad.

Lo extrañaremos mucho, pero sin duda, estará por siempre dentro de nosotros.

José M. Ceriani Cernadas 
ANEXO

\section{CUESTIONARIO}

Esta encuesta se preparó para medir los niveles de conocimiento entre los profesionales de la salud acerca de la lactancia y la leche materna. Es posible que no conozca la respuesta a algunas preguntas; no se preocupe. Esta encuesta no está diseñada para medir su conocimiento, sino para evaluar la necesidad de capacitación en esta área y determinar los temas a incluir. Su información será confidencial. Le agradecemos su participación.

Dra. Aylin BAYDAR ARTANTAȘ (0 31229125 25/3642)

Me han informado sobre el objetivo de esta encuesta. El entrevistador ha contestado todas mis preguntas sobre la encuesta. Puedo dejar el estudio en cualquier momento, con o sin motivo. El investigador podría excluirme del estudio en contra de mi voluntad. Estoy al tanto de que podré conocer los resultados del estudio, y para ello debo llamar al número de teléfono que figura anteriormente.

\begin{tabular}{|lll|}
\hline Acepto $\square$ & No acepto $\square$ & participar en el estudio. \\
\hline
\end{tabular}

1- Edad:

2-Sexo: $\square$ Femenino $\square$ Masculino

3- Su profesión:

“* Especialista en ginecología y obstetricia

* Residente de ginecología y obstetricia

* Especialista en pediatría

" Residente de pediatría

** Especialista en medicina general

". Residente de medicina general

.* Médico de cabecera

*. Enfermera/partera

- Otra.

4- Su lugar de trabajo:

$\begin{array}{lll}\square \text { Hospital universitario } & \square \text { Hospital privado } & \square \text { CSMIPF* } \\ \square \text { Hospital de formación e investigación } & \square \text { Centro de medicina general } & \square \text { Otro........ } \\ \square \text { Hospital público } & \square \text { Centro de salud público } \\ \text { *Centro de Salud Maternoinfantil y Planificación Familiar }\end{array}$

5- ¿Cuántos años lleva trabajando en su profesión?

6- ¿Tiene hijos? $\square$ Sí $\quad \square$ No (Si la respuesta es no, pase a la pregunta 9) 
7- Seleccione el casillero correspondiente (complete las respuestas para sus últimos cuatro hijos).

\begin{tabular}{|c|c|c|c|}
\hline & Método de parto & Estado en relación con la lactancia & \\
\hline 1. Niño & $\begin{array}{l}\square \text { Parto normal } \\
\square \text { Cesárea }\end{array}$ & $\begin{array}{l}\square \text { Nunca lo amamantó } \\
\square \text { Lo amamantó menos de } 6 \text { meses } \\
\square \text { Lo amamantó entre } 6 \text { meses y } 1 \text { añ }\end{array}$ & $\begin{array}{l}\square \text { Lo amamantó entre } 1 \text { y } 2 \text { años } \\
\square \text { Lo amamantó más de } 2 \text { años }\end{array}$ \\
\hline 2. Niño & $\begin{array}{l}\square \text { Parto normal } \\
\square \text { Cesárea }\end{array}$ & $\begin{array}{l}\square \text { Nunca lo amamantó } \\
\square \text { Lo amamantó menos de } 6 \text { meses } \\
\square \text { Lo amamantó entre } 6 \text { meses y } 1 \text { añ }\end{array}$ & $\begin{array}{l}\square \text { Lo amamantó entre } 1 \text { y } 2 \text { años } \\
\square \text { Lo amamantó más de } 2 \text { años } \\
\end{array}$ \\
\hline 3. Niño & $\begin{array}{l}\square \text { Parto normal } \\
\square \text { Cesárea }\end{array}$ & $\begin{array}{l}\square \text { Nunca lo amamantó } \\
\square \text { Lo amamantó menos de } 6 \text { meses } \\
\square \text { Lo amamantó entre } 6 \text { meses y } 1 \text { añ }\end{array}$ & $\begin{array}{l}\square \text { Lo amamantó entre } 1 \text { y } 2 \text { años } \\
\square \text { Lo amamantó más de } 2 \text { años } \\
\end{array}$ \\
\hline 4. Niño & $\begin{array}{l}\square \text { Parto normal } \\
\square \text { Cesárea }\end{array}$ & $\begin{array}{l}\square \text { Nunca lo amamantó } \\
\square \text { Lo amamantó menos de } 6 \text { meses } \\
\square \text { Lo amamantó entre } 6 \text { meses y } 1 \text { a }\end{array}$ & $\begin{array}{l}\square \text { Lo amamantó entre } 1 \text { y } 2 \text { años } \\
\square \text { Lo amamantó más de } 2 \text { años } \\
\text { o }\end{array}$ \\
\hline
\end{tabular}

8- ¿Alguna vez usó mamadera o chupete con alguno de sus hijos?

$$
\square \text { Sí } \quad \square \text { No }
$$

Preguntas 9 a 12: Seleccione la afirmación que considere verdadera (Puede seleccionar más de una)

9- ¿Cuál de las siguientes pautas se recomienda para una generación saludable?

$\square$ Lactancia materna exclusiva durante los primeros seis meses, continuar con la lactancia hasta el año junto con alimentación complementaria.

$\square$ Lactancia materna exclusiva durante los primeros seis meses, continuar con la lactancia hasta los dos años junto con alimentación complementaria.

$\square$ Lactancia materna exclusiva durante los primeros cuatro meses, continuar con la lactancia hasta los dos años junto con alimentación complementaria y leche maternizada.

$\square$ Lactancia materna exclusiva durante los primeros cuatro meses, continuar con la lactancia hasta los dos años junto con alimentación complementaria.

10- ¿El riesgo de cuáles de las siguientes enfermedades se reduce con la lactancia materna?

\begin{tabular}{|lll|}
\hline$\square$ Otitis & $\square$ Invaginación & $\square$ DM tipo 2 \\
$\square$ Alergias & $\square$ Estenosis pilórica & $\square$ Asma \\
$\square$ Apendicitis & $\square$ Obesidad & \\
\hline
\end{tabular}

11- ¿Contra cuáles de las siguientes enfermedades protege la lactancia materna?

\begin{tabular}{|ll|}
\hline$\square$ Cáncer de mama & $\square$ Osteoporosis \\
$\square$ Cáncer de ovario & $\square$ Anemia \\
\hline
\end{tabular}


12- ¿En qué casos está contraindicada la leche materna?

\begin{tabular}{|lll|}
\hline$\square$ VHLT-1 & $\square$ Hepatitis A & $\square$ CMV \\
$\square$ VIH & $\square$ Hepatitis B & $\square$ Mastitis \\
$\square$ Tuberculosis activa & $\square$ Hepatitis C & \\
\hline
\end{tabular}

Seleccione verdadero o falso con respecto a las siguientes declaraciones. Si no está seguro, o si desconoce la respuesta, seleccione "no sabe".

13- El Ministerio de Salud recomienda iniciar la lactancia materna en el término de 30 minutos/1 hora después del parto.
$\square$ Verdadero
Falso
$\square$ No sabe

14- El calostro contiene niveles elevados de zinc y vitaminas A, D y B12.
$\square$ Verdadero
Falso
$\square$ No sabe

15- Los recién nacidos pierden entre el $7 \%$ y el $10 \%$ de su peso durante la primera semana de vida, aunque se alimenten con leche materna.
$\square$ Verdadero
Falso
No sabe

16- Si un recién nacido aumenta menos de 500 gramos en un mes u orina menos de seis veces al día con un olor muy fuerte, indica que el recién nacido no consume suficiente leche materna.
$\square$ Verdadero
$\square$ Falso
$\square$ No sabe

17- En el caso de los mellizos, la leche materna no es suficiente y es necesario usar leche maternizada.
$\square$ Verdadero
Falso
$\square$ No sabe

18- Es probable que se produzcan grietas en los pezones después del primer bebé.
$\square$ Verdadero
$\square$ Falso
$\square$ No sabe

19- Se debe aplicar crema desde el primer día para evitar las grietas o la irritación en los pezones.
$\square$ Verdadero
Falso
No sabe

20- Las madres con pezones planos o retraídos no pueden amamantar y necesitan masajes o accesorios para la lactancia.
$\square$ Verdadero
Falso
$\square$ No sabe

21- En los casos de obstrucción mamaria, el pecho debe descansar un momento.
$\square$ Verdadero
$\square$ Falso
$\square$ No sabe 
22- El uso de mamaderas o chupetes hace que el bebé rechace el pecho.
$\square$ Verdadero
$\square$ Falso
$\square$ No sabe

23- La extracción de leche manual no es tan buena como la extracción con sacaleches.
$\square$ Verdadero
$\square$ Falso
$\square$ No sabe

24- La leche congelada debe descongelarse a temperatura ambiente antes de dársela al bebé.
$\square$ Verdadero
$\square$ Falso
$\square$ No sabe

25- ¿Brinda información sobre la lactancia materna en su práctica diaria?
$\square$ Sí
$\square$ No

26- ¿Recibió capacitación sobre la lactancia y la leche materna?
$\square$ Sí
$\square$ No (Si la respuesta es no, pase a la pregunta 29)

27- Si recibió capacitación, ¿cuándo fue?

$\square$ Durante la carrera o residencia

$\square$ En el lugar de trabajo

$\square$ De profesionales de la salud en el hospital donde nació mi bebé

$\square$ De los profesionales de la salud durante los controles prenatales

$\square$ En otro momento...

28- ¿Cuánto tiempo ha transcurrido desde que recibió capacitación?

$\square$ Menos de 2 años

$\square$ Entre 2 y 5 años

$\square$ Entre 5 y 8 años

$\square$ Más de 8 años

29- ¿Considera que necesita capacitación sobre lactancia y leche materna?

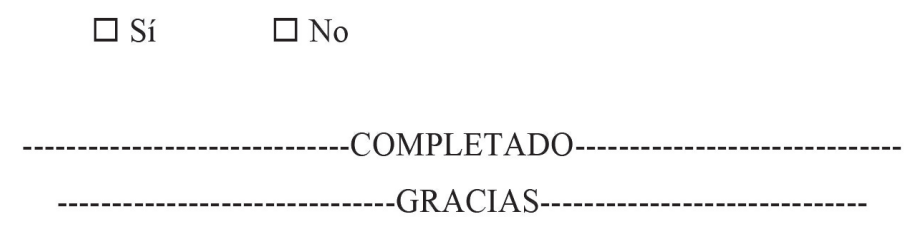

